\title{
PESQUISA DE HEMOGLOBINAS VARIANTES NA POPULAÇÃO DE FRANCISCO BELTRÃO - PARANÁ
}

\author{
SEARCH OF VARIANT HEMOGLOBINS IN THE POPULATION OF \\ FRANCISCO BELTRÃO - PARANÁ
}

\author{
Da Silva, A.C.P. ${ }^{1}$, Piva, R.D. ${ }^{2}$, Hofmann, G.G. ${ }^{2}$, Maffessoni, T. ${ }^{2}$, Velasquez, P.A.G. \\ Afiliações: 1- Farmacêutica do Curso de Farmácia da Universidade Paranaense - UNIPAR; 2- Discentes do Curso de Farmácia da \\ Universidade Paranaense - UNIPAR; 3 - Docente do Curso de Farmácia da Universidade Paranaense - UNIPAR. \\ Avenida Júlio Assis Cavalheiro, 2000, Bairro Industrial, Francisco Beltrão, Paraná, Brasil. CEP: 85601-000. patigurgel@ prof.unipar.br.
}

\section{Resumo}

As hemoglobinopatias constituem um grupo de alterações autossômicas, na maioria das vezes recessivas, caracterizadas pela produção de hemoglobinas (Hb) estruturalmente anormais ou pelo desequilíbrio no ritmo de síntese das cadeias globínicas. Considerando que todas essas hemoglobinopatias determinam importantes manifestações clínicas, cabe ressaltar a importância do diagnóstico laboratorial precoce destas patologias, evitando as consequências deletérias da doença. Diante do exposto o presente trabalho teve como objetivo pesquisar hemoglobinas variantes na população de Francisco Beltrão, Paraná. Pacientes com pedido médico de hemograma, atendidos nos serviços de um Laboratório de Análises Clínicas (LAC) de Francisco Beltrão foram abordados após a coleta de sangue. Os objetivos do trabalho foram expostos, bem como a metodologia. O sangue foi colhido conforme protocolo do laboratório, por punção venosa com EDTA como anticoagulante. Após a utilização das amostras pelo laboratório, estas foram encaminhadas para laboratório de apoio para realização da eletroforese de hemoglobina e os resultados do hemograma foram repassados pelo LAC. Cinquenta e dois pacientes fizeram parte da pesquisa, no entanto nenhum apresentou alteração na composição hemoglobínica. Ressalta-se a importância de prosseguir a pesquisa na região em um grupo maior de pessoas focando naqueles com anemia ou alterações hematimétricas uma vez que as características miscigenatórias corroboram a existência de hemoglobinas variantes no local.

Palavras-chave: Hemoglobinas; hemoglobinopatias; talassemias; hemoglobina falciforme; anemia.

\section{Abstract}

Hemoglobinopathies are a group of autosomal changes, most often recessive, characterized by the production of structurally abnormal hemoglobins $(\mathrm{Hb})$ or by the imbalance in the synthesis rate of globin chains. Considering that all these hemoglobinopathies determine important clinical manifestations, it is important to emphasize the importance of early laboratory diagnosis of these pathologies, avoiding the deleterious consequences of the disease. Given the above, the present work aimed to research variant hemoglobins in the population of Francisco Beltrão, Paraná. Patients with a medical blood count who were treated at the services of a Francisco Beltrão Clinical Analysis Laboratory (LAC) were approached after blood collection. The objectives of the work were exposed, as well as the methodology. Blood was collected according to laboratory protocol by venous puncture with EDTA as anticoagulant. After the samples were used by the laboratory, they were sent to the support laboratory for hemoglobin electrophoresis and the blood count results were passed through the LAC. Fifty-two patients were part of the research, however none showed alteration in hemoglobin composition. We emphasize the importance of continuing the research in the region in a larger group of people focusing on those with anemia or hematimetric changes since the miscegenatory characteristics corroborate the existence of variant hemoglobins in the site.

Keywords: Hemoglobins; hemoglobinopathies; thalassemia; sickle cell hemoglobin; anemia 


\section{Introdução}

A hemoglobina humana $(\mathrm{Hb})$ é uma molécula globular composta de dois pares de cadeias globínicas polipeptídicas, sendo um par de cadeias do tipo alfa $(\alpha)$ e um par de cadeias do tipo beta $(\beta) .{ }^{1}$ Cada cadeia tem sua estrutura unida quimicamente a um grupo prostético heme, que se liga reversivelmente à molécula de oxigênio $\left(\mathrm{O}_{2}\right)$, cumprindo assim a função primária da $\mathrm{Hb}$, que é o transporte de $\mathrm{O}_{2}$ dos pulmões para os tecidos periféricos. ${ }^{2}$

O sangue de um adulto, normalmente, contém três tipos de hemoglobinas, a $\mathrm{HbA}, \mathrm{HbA}_{2}$ e Hb Fetal (HbF). A HbA é a forma mais comum e abundante, correspondendo a cerca de $96 \%$ da $\mathrm{Hb}$ total de um indivíduo, formada por duas cadeias alfa e duas cadeias beta, as demais hemoglobinas normais humanas, correspondem a uma pequena parcela do total de $\mathrm{Hb}$ de um adulto. A $\mathrm{HbA}_{2}$ composta de duas cadeias proteicas alfa e duas delta ( $\delta)$, representa de 2 a $3,7 \%$ da quantidade total de $\mathrm{Hb}$, enquanto a $\mathrm{Hb}$ Fetal ( $\mathrm{HbF})$ formada por duas cadeias alfa e duas cadeias gama $(\gamma)$ representa de 0 a $1,5 \%$, das $\mathrm{Hb}$ circulantes. ${ }^{3,4}$

Os defeitos genéticos das hemoglobinas, denominados hemoglobinopatias, constituem um grupo de alterações autossômicas, na maioria das vezes recessivas e são as doenças genéticas mais comuns em todo o mundo, com mais de 1.000 diferentes alelos mutantes caracterizados a nível molecular. As hemoglobinopatias ocorrem quando alterações genéticas mudam a sequência de aminoácidos das cadeias globínicas, ocasionando a produção de hemoglobinas variantes, $(\mathrm{HbC}$ e $\mathrm{HbS})$, alterações quantitativas das cadeias polipeptídicas das globinas (talassemias), ou ainda, persistência hereditária da hemoglobina fetal. Essas mutações além de alterar a estrutura e função das hemoglobinas, também faz com que a sobrevida das hemácias seja menor, ocasionando uma anemia crônica. ${ }^{4,5}$

Segundo a Organização Mundial da Saúde (OMS), as hemoglobinopatias são um problema de saúde crescente e possuem alta taxa de morbidade, principalmente em países de baixa e média renda. ${ }^{6,7}$ Cerca de 270 milhões de pessoas são afetadas em todo o mundo e aproximadamente $15 \%$ da população é portadora assintomática de formas de anemias hereditárias. ${ }^{7,8}$

$$
\text { Diversos estudos indicam que as }
$$
primeiras mutações nas moléculas de $\mathrm{Hb}$ apareceram no continente africano. No Brasil, a introdução das hemoglobinopatias ocorreu pela imigração dos escravos africanos e subsequente mistura racial entre os diferentes grupos populacionais, a população brasileira apresenta 
diversificados graus de miscigenação e grande heterogeneidade genética, o que contribui para a distribuição de genes anormais das globinas inerentes aos grupos étnicos de uma população. ${ }^{9}$

$$
\text { A miscigenação influenciou na }
$$
dispersão de doenças como as talassemias e a anemia falciforme. As talassemias afetam com maior frequência descendentes asiáticos e europeus; desta forma essas doenças são frequentes em locais onde houve colonização por esses grupos, especialmente italianos. ${ }^{10}$ No Brasil, a maior atenção é voltada para a detecção da Hemoglobina S, Hemoglobina $\mathrm{C}$ e a betatalassemia. As duas primeiras apresentam uma importância nacional pela sua alta frequência entre afrodescendentes. Já a beta-talassemia possui importância regional, uma vez que afeta mais a população com descendência italiana. Mas de acordo com dados da OMS, essas três hemoglobinopatias são suficientes para causar um alto grau de morbimortalidade no Brasil., ${ }^{71}$

$$
\text { O grau de anemia causado pela doença é }
$$

variável. Em casos de homozigose, o fenótipo é clinicamente significante e geralmente resulta em anemias graves, enquanto na heterozigose, os portadores podem geralmente são assintomáticos. Considerando o seu caráter hereditário e que todas essas hemoglobinopatias determinam importantes manifestações clínicas, cabe ressaltar a importância do diagnóstico laboratorial precoce, bem como o aconselhamento genético, que pode reduzir de forma significativa a frequência e complicações dessa doenças. ${ }^{6,7}$

Os estudos populacionais permitem a detecção de heterozigotos e o aconselhamento genético, evitando as consequências deletérias da doença com consequente melhoria na qualidade de vida dos doentes, dessa forma, esse estudo teve por objetivo pesquisar a presença de hemoglobinas variantes na população de Francisco Beltrão - Paraná.

\section{Métodos}

Após aprovação do Comitê de Ética em Pesquisa com Seres Humanos (Parecer $\mathrm{n}^{\circ}$. 3.179.660/2019), pacientes atendidos nos serviços de um Laboratório de Análises Clínicas da cidade de Francisco Beltrão durante o $1^{\circ}$ semestre de 2019, foram abordados após a coleta de sangue.

Todos os pacientes com pedido médico de hemograma foram incluídos na pesquisa. Durante a abordagem os objetivos da pesquisa foram expostos aos pacientes, bem como a sua metodologia. Para os pacientes que aceitaram participar do estudo foi solicitado que o mesmo assinasse o Termo de Consentimento Livre e 
Esclarecido e dados como nome, endereço, idade, sexo e casos familiares de anemia foram obtidos desses pacientes por meio de uma entrevista.

$$
\text { O sangue foi colhido conforme }
$$

protocolo do laboratório, por punção venosa com

EDTA como anticoagulante e após a utilização das amostras pelo laboratório, as amostras foram encaminhadas para laboratório de apoio para realização de eletroforese de hemoglobina (por HPLC), a fim de identificar a presença de hemoglobinas variantes e a frequência das hemoglobinas normais em relação ao total de hemoglobinas circulantes. Os resultados do hemograma que incluem contagem de eritrócitos, hemoglobina, VCM, HCM e RDW foram repassados pelo laboratório.

\section{Resultados}

Um total de 52 pacientes participou do estudo, sendo que todos apresentaram a eletroforese de hemoglobina dentro da normalidade. As respostas ao questionário podem ser visualizadas na Tabela 1 . Já os resultados dos demais parâmetros avaliados encontram-se na Tabela 2.

Tabela 1: Frequência das respostas dadas pelos pacientes atendidos em um Laboratório de Análises Clínicas no $1^{\circ}$ semestre de 2019, durante a entrevista.

\begin{tabular}{rr}
\hline Variáveis & Valor absoluto \\
& $(\%)$ \\
\hline
\end{tabular}

\begin{tabular}{cr}
\hline Sexo & $30(57,7)$ \\
Feminino & $22(42,3)$ \\
Masculino & $17(32,7)$ \\
Faixa etária dos pacientes & $26(50,0)$ \\
Idoso ( $\geq$ a 60 anos) & $08(15,4)$ \\
Adulto (30 a 59 anos) & $00(00)$ \\
Jovem (18 a 29 anos) & $01(1,90)$ \\
Adolescente (12 a 17 anos) & \\
Criança (até 11 anos) & $09(17,3)$ \\
Já teve anemia alguma vez? & Sim \\
Não & $43(82,7)$ \\
Alguém na família já teve & \\
anemia? $\quad$ Sim & $07(13,5)$ \\
Não & $45(86,5)$ \\
\hline
\end{tabular}

Fonte: dados da pesquisa.

Tabela 2: Resultados dos exames dos pacientes atendidos em um Laboratório de Análises Clínicas no $1^{\circ}$ semestre de 2019 na cidade de Francisco Beltrão, Paraná.

\begin{tabular}{|c|c|}
\hline Exames & Resultados (\%) \\
\hline \multicolumn{2}{|l|}{ Eritrócitos } \\
\hline Normal & $49(94,2)$ \\
\hline Diminuídos & $03(5,8)$ \\
\hline \multicolumn{2}{|l|}{ Hemoglobina } \\
\hline Normal & $43(82,7)$ \\
\hline Diminuída & $01(1,9)$ \\
\hline Aumentada & $08(15,4)$ \\
\hline \multicolumn{2}{|l|}{ Hematócrito } \\
\hline Normal & $45(86,5)$ \\
\hline Diminuído & $03(5,8)$ \\
\hline Aumentado & $04(7,7)$ \\
\hline \multicolumn{2}{|l|}{ VCM } \\
\hline Normal & $49(94,2)$ \\
\hline Diminuído & $02(3,8)$ \\
\hline Aumentado & $01(1,9)$ \\
\hline \multicolumn{2}{|l|}{ HCM } \\
\hline Normal & $37(71,2)$ \\
\hline Diminuído & $02(3,8)$ \\
\hline Aumentado & $13(25,0)$ \\
\hline \multicolumn{2}{|l|}{ RDW } \\
\hline Normal & $51(98,1)$ \\
\hline Aumentado & $01(1,9)$ \\
\hline
\end{tabular}

Fonte: dados da pesquisa.

\section{Discussão}

Os estudos de incidência de $\mathrm{Hb}$ variantes de uma determinada população são fundamentais para verificar a ocorrência e os potencias fatores de risco dessas doenças, além de fornecerem um panorama dos programas de triagem e os riscos 
aos quais a população de cada região está submetida, em especial para as formas homozigotas, nas quais a intervenção clínica precoce favorece a melhoria na qualidade de vida do paciente. A HbS é a hemoglobina variante mais incidente no Brasil, ressaltando a importância do seu diagnóstico mesmo em portadores assintomáticos, no entanto, nas regiões Sul e Sudeste foi observado $1,9 \%$ de hemoglobinopatias, com predomínio de talassemias, devido à forte colonização e descendência europeia dessas regiões ${ }^{12,13}$. Em Francisco Beltrão e região, a partir de 1940 houve expressiva migração por parte de descendentes europeus, principalmente italianos, alemães e poloneses, sendo que atualmente a população continua, em sua grande maioria, de influência europeia $^{14}$.

No presente estudo não foi detectada a presença de hemoglobinas variantes nos pacientes analisados. Esse resultado negativo pode ser explicado pelo número limitado de participantes na pesquisa, além disso, esse estudo não foi direcionado a pacientes que já apresentavam algum tipo de anemia ou qualquer outra alteração no hemograma. Além disso, após a realização dos exames apenas um paciente apresentou-se com anemia (Hb abaixo dos valores de referência).
Pensando nisso, novos estudos serão realizados, utilizando uma maior população amostral e restrita a pacientes com alterações hematológicas.

Na região Sul e Sudeste do Brasil há elevado número de descendentes europeus, e consequente alta incidência de anemias microcíticas com níveis de ferro sanguíneo normal ou elevado, sugestivas de talassemia. Para diferenciá-las das demais hemoglobinopatias é de extrema importância a realização do exame de eletroforese de hemoglobina. Porém, ainda existem algumas dificuldades e limitações para a detecção de hemoglobinas variantes, uma vez que se faz necessário o conhecimento da história clínica do paciente, para melhor elucidação diagnóstica bem como histórico familiar e a possibilidade de realização do exame em parentes de primeiro grau, além de informações relativas ao eritograma e seus índices hematimétricos ${ }^{15,16}$.

Dentre as formas heterozigotas de hemoglobinopatias, foi constatado que a maior dificuldade diagnóstica está sobre as formas talassêmicas que mesmo com kits com grande sensibilidade e metodologia de fácil realização, as incertezas para finalizar o diagnóstico permanecem. Nesses casos é importante a utilização de técnicas seletivas de confirmação, aplicadas de forma individualizada e aliadas ao 
histórico clinico com dados hematológicos prévios ${ }^{13,17}$.

O traço talassêmico $\alpha+$ heterozigoto ($\alpha / \alpha \alpha$ ), é uma forma de talassemia assintomática e com alterações laboratoriais mínimas ou ausentes, o que dificulta o seu diagnóstico por técnicas laboratoriais convencionais ${ }^{18}$. Em 2001, Borges e colaboradores $^{19}$, estudaram a relação entre pacientes com microcitose e hipocromia sem anemia e relataram a correlação com a $\alpha$ talassemia. Em seu estudo, 49,9\% dos casos analisados possuíam $\alpha$-talassemia, $42,8 \%$ eram indivíduos heterozigotos e 5,3\% homozigotos. Esses dados são de total importância clínica, uma vez que a maioria dos pacientes são tratados com reposição oral de ferro desnecessariamente. O estudo ainda descreve que os negros com VCM e HCM baixo tem $70,4 \%$ de chance de possuir a doença. Em caucasianos a chance é de 41,5\%. Considerando o alto nível de miscigenação no território brasileiro, a prevalência pode ser relativamente alta. No presente estudo observouse um paciente com microcitose sem anemia e outro com hipocromia isolada. No entanto como os demais dados, inclusive a eletroforese, foram normais descartou-se qualquer outra discussão.

As variantes de hemoglobina $\mathrm{S}$ e $\mathrm{C}$ também apresentam dificuldade de caracterização, a eletroforese em $\mathrm{pH}$ ácido pode ser utilizada como teste de confirmação e servir de suporte para uma elucidação diagnóstica mais segura. O maior problema está no diagnóstico das variantes raras que requerem prática do analista e conhecimento de metodologias específicas para a confirmação dos resultados ${ }^{12,20}$.

\section{Conclusão}

Não se observou portadores de hemoglobinopatias dentre os 52 pacientes estudados em Francisco Beltrão - Paraná durante o ano de 2019. Pretende-se ampliar este estudo para uma população maior focando a pesquisa para o grupo de pacientes que já se apresente com dados hemoglobínicos e/ou hematimétricos alterados, uma vez que a região possui características miscigenatórias que corroboram a busca por essas hemoglobinas variantes.

\section{Referências}

1. Claudino GNG, Pitombeira MS. Aspectos moleculares da anemia falciforme. J. Bras. Patol. Med. 2003; 39(1):51-56.

2. Wenning M, Sonati M. Hemoglobinopatias Hereditárias. São Paulo: Manole; 2007. 
3. Zago MA. Estrutura, síntese e genética das hemoglobinas. São Paulo: Editora Atheneu; 2013.

4. Hoffbrand AV. Fundamentos da Hematologia. $6^{\circ}$ ed. Porto Alegre: Artmed, 2013.

5. Madigan C, Malik P. Pathophysiology and therapy for haemoglobinopathies. Part I: sickle cell disease. Expert Rev Mol Med. 2006; 8(9):1-23.

6. Orlando GM, Naoum PC, Siqueira FAM, Bonini-Domingos CR. Diagnóstico laboratorial de hemoglobinopatias em populações diferenciadas. Rev Bras de Hemato e Hemo. 2000; 22(2): 111-121.

7. Angastiniotis M, Corrons JLV, Soteriades ES, Eleftheriou A.The Impact of Migrations on the Health Services for Rare Diseases in Europe: The Example of Haemoglobin Disorders. The Scientific World Journal. 2013; 2013(3):727905.

8. Leoneli GG, Imperial RE, Marchi-Salvador DP, Naoum PC, Bonini-Domingos CR. Hemoglobinas anormais e dificuldade diagnóstica. Rev Bras de Hemato e Hemo. 2000; 22(3): 396-403.

9. Oliveira JB, Moraes KC. Hemoglobinopatias: uma questão de saúde pública revisão bibliográfica. In: XIII Encontro Latino Americano de Iniciação Científica; IX Encontro Latino Americano de PósGraduação; III Encontro Latino Americano de Iniciação Cientifica Júnior; 2009 out 16-17. Vale do Paraíba (SP): Universidade do Vale do Paraíba. Disponível em: http://www.inicepg.univap.br/cd/INIC_2 009/anais/arquivos/RE_0684_0718_02.pdf

10. Lisot C, Silla L. Screening for hemoglobinopathies in blood donors from Caxias do Sul, Rio Grande do Sul, Brazil: prevalence in an Italian colony. Cad Saude Publica. 2004; 20(6):1595-1601.

11. Sales RL, Soares AP, Moita Neto JM, Costa RS, Rocha SS, Nogueira LT. Analysis of blood quality screening in neonatal. J Nurs UFPE on line. 2015; 9(2):677-82.
12. Ivo LM, Araujo RMO, Barbieri RA, Corrêa Filho RAC, Pontes ERJC, Botelho CAO. Scope and efficiency of the newborn screening program in identifying hemoglobin S. Revista Brasileira de Hematologia e Hemoterapia. 2014; 36(1):14-18.

13. Barros SAF, Assunção BR, Santos CCD. Anemia falciforme: uma revisão acerca da doença, novos métodos diagnósticos e tratamento. Revista Eletrônica Acervo Saúde. 2017; 9(1):856-863.

14. Briskievicz, M. Territorialidade e identidade: a migração de descendentes de italianos no município de Francisco Beltrão - Paraná. [Dissertação]. Francisco Beltrão: Universidade Estadual do Oeste do Paraná; 2012.

15. Verrastro T, Lorenzi Tf, Wendel Neto, S. Hematologia e Hemoterapia: Fundamentos de Morfologia, Fisiologia, Patologia e Clínica. 1.ed. São Paulo: Atheneu. 2005.

16. Naoum PC. Eletroforeses: hemoglobinopatias, proteínas séricas, lipoproteínas, DNA. São Paulo: Editora Santos, 2012.

17. Almeida LP, Wengerkievicz AC, Viviani NM, ADM, Mendes ME, Sumita NM. O laboratório clínico na investigação dos distúrbios da hemoglobina. J. Bras. Patol. Med. 2011; 47(3):271-278.

18. Hoffbrand AV, Moss PAH. Fundamentos em hematologia. 6. ed. Porto Alegre: ARTIMED, 2013.

19. Borges E, Wenning MRSC, Kimura EM, Gervásio SA, Costa FF, Sonati M.F.High prevalence of alpha-thalassemia among individuals with microcytosis and hypochromia without anemia. Braz J Med Biol Res. 2001; 34(6):759-762.

20. Nogueira KDA, Silva WDL, Paiva SG. Diagnóstico laboratorial da anemia falciforme. Rev. Científicado ITPAC. 2013; $6(4): 1-5$

Data de submissão: 23/11/2019 Data de aprovação: 09/03/2020 